Vol. 01, No. 02, abril - junio 2021, 01-14

\title{
Estudio de tambos de Uruguay con diferentes sistemas de producción, gestión y biotipos. Análisis de casos
}

\section{Study of dairy farms in Uruguay with different production systems, management and biotypes. Case analysis}

\author{
Esteban Krall1,a* , Pedro Arbeletche ${ }^{2, b}$, Marcelo Gurin ${ }^{1, c}$ \\ ${ }^{1}$ Polo Educativo Tecnológico de Paysandú, Universidad Tecnológica, Paysandú, Uruguay. \\ ${ }^{2}$ Departamento de Ciencias Sociales, Facultad de Agronomía (Udelar), Paysandú, Uruguay.

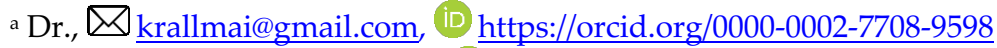 \\ b Ing., $\bigotimes_{\text {arbe19@fagro.edu.uy, (D) http://orcid.org/0000-0001-8929-4356 }}$ \\ cTecnólogo Químico, $\bigotimes_{\text {marcelo.gurin@utec.edu.uy (iD https://orcid.org/0000-0002-2735-0196 }}$ \\ * Autor de Correspondencia: Tel. +59 892067984 \\ http://dx.doi.org/10.25127/riagrop.20212.674
}

http://revistas.untrm.edu.pe/index.php/RIAGROP revista.riagrop@untrm.edu.pe

Recepción: 18 de enero 2021

Aprobación: 22 de marzo 2021

Este trabajo tiene licencia de Creative Commons. Attribution-NonCommercial-ShareAlike $\quad 4.0$ International Public License - CC-BY-NC-SA 4.0

\section{Resumen}

Dada la difícil coyuntura de la lechería exportadora uruguaya, en un marco internacional de precios inciertos, Se hace necesarios estudios que contribuyan a orientar sobre opciones de sistemas y biotipos, que apunten a fortalecer la sobrevivencia de los tambos. Para esto, las variables productivas deben ser medidas con precisión y miradas en conjunto con las económicas. Se estudiaron seis tambos variables productivas (estado corporal al parto y lactancia, peso vivo, producción de leche y diaria de grasa y proteína y en relación al peso, porcentaje de grasa y proteína) y cuatro variables económicas (ingreso neto, costos variables y funcionamiento, margen de alimentación). Los biotipos utilizados son holando, jersey y cruzas entre estas dos razas y con roja sueca. En el análisis estadístico, se realizaron estudios de correlación y test de Tuckey para comparación de medias. Se diferencian predios con opciones de mayor y menor gasto, producción y resultado económico. Los de menor gasto optan por biotipos más rústicos. El holando pareció necesitar más las reservas corporales para la producción. 
La producción de leche y sólidos diarios fue mayor en las cruzas pero el porcentaje y producción de sólidos en relación al peso fue mayor en jersey.

Palabras clave: Biotipos, margen económico, producción lechera.

\begin{abstract}
Given the difficult situation of Uruguayan dairy exports in an international context of uncertain prices, studies that contribute to guide on options of systems and biotypes, which aim to strengthen the survival of the dairy farms seem useful. For this, the productive variables must be measured with precision and looked at in conjunction with the economic ones. We studied in six dairy farms, six production variables (body state at calving and lactation, live weight, milk production and daily fat and protein and in relation to weight, fat percentage and protein) and in four economic variables (net income, variable costs and operation, feed allowance). The biotypes used are Holstein, Jersey and crosses between these two breeds and with Swedish red. In the statistical analysis, correlation studies and Tuckey's test were performed to compare means. We differentiate farms with options of higher and lower expenditure, production and economic result; those with lower expenditure opt for more rustic biotypes. The Holstein seemed to need more body reserves for the production; the daily production of milk and solids was greater in the crosses but the percentage and production of solids in relation to the weight was greater in Jersey.
\end{abstract}

Keywords: Biotypes, economic margin, dairy production.

\section{INTRODUCCIÓN}

Desde el año 2013, se evidencia un marcado descenso en el precio de la leche a nivel internacional. A pesar de haber tenido una leve recuperación a partir del año 2017, no se han logrado los niveles de la década anterior (Bartaburu y Cesar, 2016; INALE, 2020). Esta situación ha llevado a los tambos a extremar la búsqueda de la eficiencia del sistema productivo (leche, carne, agricultura) como forma de sobrevivir en la producción. El abandono de la producción y la desaparición de tambos es un proceso de larga data en Uruguay, en el año 2020, existe 2321 remitentes, un $30 \%$ menos que los registrados 10 años antes (DIEA, 2020; INALE, 2020; Rosa y Arbeletche, 2016). Según los analistas $\mathrm{y}$ las gremiales de productores, una gran proporción de los tambos se encuentran con resultados económicos negativos en los últimos años (ANPL, citado por Diario La Mañana, mayo, 2020).
A pesar de esta situación en la base productiva, la remisión total a planta estuvo en constante crecimiento hasta el año 2014. Luego se mantuvo estancada en alrededor de los 2000 millones de litros hasta la actualidad (INALE, 2020).

Para algunos productores, esta búsqueda de eficiencia económica implica realizar continuamente acciones para reducir los costos productivos. Esto atenta, en muchas ocasiones, la expresión del potencial productivo del ganado. El principal argumento es que logrando reducir los costos, pese a obtener una menor productividad física, se contribuye a sustentar el sistema. Para ello, muchas veces, se busca utilizar suplementos que son subproductos de la agroindustria local, que no necesariamente son los mejores, por su calidad, en la producción de litros de leche por vaca. Otra práctica para la reducción de costos es utilizar el campo natural de bajo costo en una etapa del ciclo productivo (período seco). Esto 
último va acompañado de cambios en el biotipo elegido, se opta por seleccionar los más rústicos.

Sin embargo, y contrastando con estas alternativas de disminución de costos, hay muchos productores que entienden, por ejemplo, que el aumento del gasto de la alimentación es la forma de sostener el sistema a largo plazo (ANDE, 2020). En muchos casos, quienes sostienen esta opción utilizan ganados lecheros de altos requerimientos y potencial de producción en términos de litros y sólidos.

Según la encuesta de INALE (2014), se observa, en todo el país, un uso mayoritario de raza Holando Americano Canadiense (83\%), 6 \% de Holando Neozelandés, $9 \%$ de cruzas y $2 \%$ de razas Jersey y Normando. A pesar del creciente interés en biotipos diferentes al predominante (Holando Americano Canadiense), existen pocos estudios sobre la eficiencia de diferentes grupos genéticos en el Uruguay y en particular en la región litoral del país (Krall et al., 2018).

Por tanto, la evaluación (productiva y económica) de distintas opciones de sistemas de producción prediales, parece necesaria para contribuir a orientar decisiones futuras. La información de tambos que incluyan algunas variables que afectan la eficiencia productiva (litros de leche, kilogramos de grasa y proteína, estado corporal, peso vivo, alimentación utilizada, reproducción, biotipo) y económica (costo y margen de alimentación, gasto de funcionamiento, superficie, dotación animal, recursos naturales y de equipos) pueden resultar útiles, aunque sean estudios de casos y no necesariamente representen las diferentes regiones productivas del país.

\section{MATERIALES Y MÉTODOS}

En este trabajo, se estudió, en el período de febrero a diciembre de 2019, la evolución productiva de animales y para el período de julio 2018 a junio 2019 y se realizó un análisis económico y estudio de los indicadores físicos.

Se registraron eventos reproductivos (partos, celos, servicios), la estrategia de manejo reproductivo (monta natural, inseminación artificial y/o combinación de ambas). Se construyeron indicadores reproductivos, tales como días entre parto y primer servicio, número de servicios utilizados por vaca $\mathrm{y}$ preñez al servicio.

Se registraron estas variables en todas, o al menos una muestra representativa, de las vacas (por ejemplo partos de otoño) y al menos en tres momentos durante la lactancia.

Las variables registradas se corresponden a:

a) Producción de leche (litros) y composición de grasa y proteína en las etapas definidas de lactancia (temprana, media y tardía). Por tanto, se utilizaron las variables producción media de leche (PL), porcentaje de grasa (Gr) y proteína $(\mathrm{Pr})$, producción diaria de grasa (KgGrD) y proteína (KgPrD). Estas dos últimas variables se relacionaron con el peso vivo. Se obtuvieron de esa manera los gramos de grasa y proteína por $\mathrm{kg}$ de peso (GrGrPV y GrPrPV respectivamente).

b) Se registró el estado corporal al parto (ECP) y en lactancia temprana y tardía (EC1, EC2 respectivamente) a partir del día 30 de la lactancia, de acuerdo a la escala 1 a 5 de Edmonsond et al. (1989). De la diferencia entre ECP y EC1, se genera la variación de estado (VAREC). 
c) Peso vivo durante la lactancia tempranamedia, que fue registrado en una balanza electrónica con aproximación al kilo.

d) Se describe el biotipo genético (madres y toros utilizados) del rodeo lechero del predio.

e) Se registró la alimentación ofrecida distinguiendo entre suplementos concentrados, pasturas ([por apreciación visual], Montossi et al, 2013) y reservas forrajeras ofrecidas.

Para evaluar la eficiencia económica se utilizaron metodologías tradicionales de gestión de empresas y se elaboraron indicadores como ingreso neto generado por establecimiento, costos de producción, se enfatizaron los costos relacionados a los elementos principales de la producción lechera tales como el margen de alimentación y se tuvo en cuenta la composición de la oferta alimenticia (pastura, concentrado, voluminosos) y su origen (propia o comprada); incluyendo los costos de mano de obra y los relacionados al funcionamiento del tambo.

El análisis estadístico de las variables productivas se planteó a través de estudios de correlación entre variables y del test de diferencias honestamente significativas de Tukey para comparar medias de las variables agrupando vacas por niveles de estado corporal al parto y biotipo.

Las variables mencionadas serán presentadas según la disponibilidad de la información obtenida en cada tambo.

En este trabajo, se utilizaron seis tambos, donde se analizaron variables de estado corporal, cinco donde se recabaron las variables productivas y cuatro donde se contó con información suficiente para el estudio económico.

Los biotipos utilizados son la raza Holando (en 3 predios), la raza Jersey (en 5 predios) y la Cruza entre las dos razas anteriores (en 2 predios) y con roja sueca o sea triple cruza HolandoxJerseyxRoja Sueca (en 1 predio).

\section{RESULTADOS Y DISCUSIÓN}

\subsection{Descripción de los predios analizados.}

En las Tablas del 1 al 4 se presenta la información de las variables descriptivas de los tambos estudiados. En primer lugar, se puede que hay una variedad importante de biotipos $y$ sistemas.

En la Tabla 1, se presentan los tipos de alimentos utilizados, biotipos, indicadores reproductivos y productivos (promedio anual del rodeo) para cada uno de los tambos analizados.

En la Tabla 2, se presentan los indicadores económicos de los predios en que fue posible el cálculo. Destacó el precio por litro de leche remitida en centavos de dólares; el costo de alimentación por litro; el costo de funcionamiento por litro y el margen bruto por litro de leche y el margen de alimentación, calculado como la diferencia entre el producto bruto restado los costos de alimentación.

En la Tabla 3, se presentan las variables productivas promedio de las vacas estudiadas de los seis tambos, es decir, la producción media de leche en los meses de lactancia, porcentaje de grasa y proteína, producción diaria de grasa y proteína, gramos de grasa y proteína por $\mathrm{kg}$ de peso, peso vivo, estado corporal al parto y en lactancia temprana $\mathrm{y}$ 
tardía y la diferencia entre ECP y EC1 o variación de estado.

Dadas las diferencias en los sistemas de producción y biotipos de los tambos, en la Tabla 4, se presentan los datos de comportamiento de las variables productivas para los tres tambos con Holando y Cruzas.

El tambo C es el de menor gasto de alimentación y menor producción (Tablas 1, 2 y 3) por opción de manejo del sistema que provoca una disminución del potencial de producción por vaca de la raza Holando (de origen nacional) en ese predio. Esta situación es visualizable tanto para el Holando como para las vacas cruza (Tabla 4). También, en este caso y por una coyuntura particular de la empresa a la cual se remite la leche, el precio recibido por litro es menor a los otros tambos (Tabla 2).
En el otro extremo del nivel de producción y también con un gasto importante, se encuentra el tambo E. En este caso hay ganado Holando de buen nivel genético (origen norteamaricano) que, además, al ser alimentado abundantemente (reciben $7 \mathrm{~kg}$ de concentrados y disponen de mucha pastura y reservas forrajeras) su nivel de producción llega a casi los 29 litros por animal y por día (Tabla 4). Se observa que la evaluación de estados corporales del productor LE, se encuentra que en el Holando siempre desciende el estado (desde ECP hasta EC2), mientras que en cambio la cruza (HolxJerx Roja Sueca) recupera en EC2 (en lactancia avanzada). Esto sugiere un mejor ajuste a los requerimientos en este predio para el biotipo cruza y que para el Holando.

Tabla 1. Variables de alimentación, biotipos, reproductivas y productivas por tambo

\begin{tabular}{|c|c|c|c|c|c|c|c|c|c|c|c|c|}
\hline Tambos & CON & PAST & RF & HOLAN & JER & CRU & DPS & SU & $\begin{array}{l}\text { LT/ } \\
\text { VOD }\end{array}$ & $\begin{array}{l}\text { HA } \\
\text { VM }\end{array}$ & $\begin{array}{c}\text { VM/ } \\
\text { HaVM }\end{array}$ & $\begin{array}{c}\text { VO } \\
\text { Promedio }\end{array}$ \\
\hline A & 6 & $\mathrm{AA}, \mathrm{VI}$ & FAR & $77 \% \mathrm{NZ}$ & $8 \% \mathrm{~N}$ & $15 \% \mathrm{~K}$ & 61 & 1.1 & 17 & 70 & 1.2 & 70 \\
\hline B & 4 & CNM,VI,PP & FAR & & NZ, NA & & -- & 1.6 & 14 & 130 & 1 & 100 \\
\hline $\mathrm{C}$ & 4 & $\mathrm{AA}, \mathrm{VI}, \mathrm{VV}$ & $\begin{array}{l}\text { SMPE, } \\
\text { FAR }\end{array}$ & $37 \% \mathrm{~N}$ & $13 \% \mathrm{~N}$ & $50 \% \mathrm{~K}$ & 92 & 1.7 & 11.3 & 70 & 1.1 & 55 \\
\hline D & 4 & VI,PP,VV & SMPE & & $\mathrm{NZ}, \mathrm{NA}$ & & 90 & 1.6 & 14.2 & 66 & 2 & 113 \\
\hline E & 7 & VI,PP & SMPE & NA $30 \%$ & & $\begin{array}{c}\text { HOLXJER } \\
\text { XRS } 70 \%\end{array}$ & 70 & 2.3 & 19.5 & 247 & 1.53 & 330 \\
\hline $\mathrm{F}$ & 4 & $\mathrm{CN}, \mathrm{PP}, \mathrm{VI}$ & -- & & NZ, NA & & 90 & 1.5 & 16 & 200 & 1 & 120 \\
\hline
\end{tabular}

\section{Referencias:}

Alimentación $=\mathrm{CON}$ (concentrados), PAST (pasturas) y RF (reservas forrajeras).

AA (alfalfa), VI (verdeo invierno), PP (pradera permanente), VV (verdeo verano), CNM (campo natural mejorado), CN (campo natural), FAR (fardo o heno), SMPE (silo maíz planta entera)

Biotipos = HOLAN (Holando), JER (Jersey) y CRU (Cruzas), NZ (neozelandés), NA (Norteamérica), N (nacional), RS (Roja sueca), K (kiwi: cruza JERx HOL)

Reproductivos = DPS (días parto-primer servicio), SU (servicios utilizados),

Productivas = LT/VOD (litros por vaca en ordeñe/día, promedio anual), Ha (superficie), HA VM (hectáreas dedicadas a la vaca masa), VM/HA VM (vaca masa (ordeñe más secas) por Ha vaca masa; VO (vacas en ordeñe promedio anual). 
Tabla 2. Precios y costos de producción por tambo

\begin{tabular}{cccccc}
\hline Tambos & Precio/I U\$S & $\begin{array}{c}\text { Costo U\$S } \\
\text { Alimentación / } ~\end{array}$ & $\begin{array}{c}\text { Costo U\$S } \\
\text { funcionamiento/I }\end{array}$ & Margen U\$S/ I & $\begin{array}{c}\text { Margen Alimentación } \\
\text { (USS/VO/día) }\end{array}$ \\
\hline A & 0.32 & 0.161 & 0.098 & 0.061 & 2.73 \\
C & 0.28 & 0.12 & 0.15 & 0.01 & 2.3 \\
D & 0.37 & 0.182 & 0.14 & 0.048 & 3.3 \\
E & 0.31 & 0.155 & 0.090 & 0.065 & 3.3 \\
\hline
\end{tabular}

Tabla 3. Estado corporal, producción y composición de la leche por tambo

\begin{tabular}{ccccccccccccc}
\hline Tambos & ECP & EC1 & EC2 & VAR EC & PESO & PL & Gr & Pr & KgGr D & KgPr D & GrGr PV & GrPr PV \\
\hline A & 2.91 & 2.81 & 2.60 & 0.10 & 498 & 16.6 & 4.00 & 3.67 & 0.67 & 0.61 & 1.43 & 1.32 \\
B & 2.92 & 2.54 & 2.59 & 0.36 & 343 & 13.9 & 4.77 & 4.03 & 0.65 & 0.56 & 1.93 & 1.68 \\
C & 2.67 & 2.70 & 2.68 & -0.11 & 465 & 11.2 & 4.63 & 3.72 & 0.50 & 0.41 & 1.12 & 0.89 \\
D & 3.13 & 2.75 & 2.60 & 0.38 & 319 & 17.0 & 5.09 & 3.86 & 0.80 & 0.61 & 2.76 & 2.09 \\
E & 3.07 & 2.58 & 2.65 & 0.40 & 540 & 24.8 & 4.32 & 3.61 & 1.06 & 0.89 & 1.98 & 1.65 \\
F & 2.60 & 2.38 & 2.24 & 0.44 & 357 & 14.1 & 4.57 & 3.77 & 0.65 & 0.54 & 1.82 & 1.49 \\
\hline
\end{tabular}

Referencias:

PL (producción media de leche), Gr (porcentaje de grasa) y Pr (proteína), KgGrD (producción diaria de grasa); KgPrD (producción diaria de proteína, GrGrPV y GrPrPV (gramos de grasa y proteína por kg de peso); PESO (peso vivo), ECP (estado corporal al parto); EC1 y EC2 (estado corporal en lactancia temprana y tardía; VAREC (diferencia entre ECP y EC1 o variación de estado)

Tabla 4. Estado corporal, producción y composición de la leche por biotipo

\begin{tabular}{lcccccccccccc}
\hline BIOTIPO & ECP & EC1 & EC2 & VAREC & PESO & PL & Gr & Pr & KgGrD & KgPrD & GrGrPV & GrPrPV \\
\hline HOLAN A & 2.62 & 2.77 & 2.57 & -0.04 & 506.18 & 16.74 & 3.97 & 3.65 & 0.68 & 0.62 & 1.41 & 1.31 \\
HOLAN C & 2.61 & 2.51 & 2.70 & 0.10 & 495.71 & 11.18 & 4.40 & 3.64 & 0.50 & 0.41 & 1.05 & 0.85 \\
HOLAN E & 2.98 & 2.58 & 2.52 & 0.44 & 596.47 & 28.94 & 3.87 & 3.41 & 1.11 & 0.99 & 1.88 & 1.66 \\
CRU A & 3.12 & 2.75 & 2.45 & 0.37 & 471.33 & 18.72 & 4.01 & 3.49 & 0.74 & 0.64 & 1.44 & 1.31 \\
CRU C & 2,73 & 2,82 & 2,77 & $-0,11$ & 461.91 & 11.73 & 4.45 & 3.66 & 0.51 & 0.43 & 1.12 & 0.92 \\
CRU E & 3.12 & 2.59 & 2.71 & 0.41 & 516.36 & 22.83 & 4.53 & 3.70 & 1.03 & 0.84 & 2.02 & 1.64 \\
\hline
\end{tabular}

Referencias: ídem Tabla 3

Los diferentes pesos vivos y producciones de leche del Holando de los tres predios observados en la Tabla 4 evidencian las diferentes genéticas y sistemas de producción. En el caso del tambo A, el mismo utiliza genética neozelandesa y logra atender los requerimientos nutricionales adecuadamente, si bien en producción de leche y estado, nos encontramos que la cruza supera levemente al
Holando. La cruza en E es de triple origen (Holando, Jersey, Roja Sueca) y el buen sistema de alimentación permite expresar un buen potencial productivo y recuperación de estado al final de la lactancia, lo que no sucede con el Holando Norteamericano. En el tambo C, el origen de las vacas, es a partir de la utilización de toros nacionales y podemos ver que las 
restricciones nutricionales afectan a ambos biotipos (Holando y cruza) por igual.

Se visualizan, además (Tablas 3 y 4), las diferencias de la raza Jersey en cuanto a tamaño (con peso vivo menor a $400 \mathrm{~kg}$ ) y porcentaje de grasa y proteína. Dentro de esta raza hay diferencias en los sistemas de alimentación: el predio $\mathrm{F}$ utiliza campo natural en el período seco, previo al parto, lo que lleva a estados corporales bajos (menor a 3 en el parto). En este último tambo, es en el que se presenta un menor ajuste de la alimentación, dado que el descenso de estado en lactancia temprana continúa en la tardía; y a pesar de esto la eficiencia reproductiva no se resiente en demasía, debido a la rusticidad de la raza. Los otros predios, por su parte tienden a recuperar estado lo que indica la existencia de un mejor ajuste nutricional.

Como se sabe, los mayores niveles de producción de leche conllevan a mayores $\mathrm{kg}$ de grasa y proteína; en este caso se observa esta situación en los predios con más litros $(\mathrm{D}, \mathrm{E})$, que son además aquellos donde es mayor la oferta de alimentos y a su vez los resultados de sólidos son también buenos (Tabla 3). Estos dos predios ( $\mathrm{D}$ y E), a pesar de tener un gasto importante en alimento, son los que logran mejor margen de alimentación por vaca y por día.

En referencia a la reproducción, todos parecen con buenos o aceptables indicadores; dos predios con Jersey ( $\mathrm{D}$ y $\mathrm{F}$ ) presentan un relativamente largo período desde el parto al primer celo (90 días); en un caso hay muy buena producción de leche y en el otro hubieron estados al parto y en lactancia un poco bajos. Los servicios utilizados fueron razonables: 1,5 por vaca. Este parámetro empeora en el predio
(E) con uso intensivo de alimentos y parte del rodeo con Holando de genética norteamericana.

Estas consideraciones (relativas al "efecto tambo") pueden afectar la comparación de biotipos de este estudio.

\subsection{Estudio de correlaciones entre las principales variables analizadas}

En las Tablas 5, 6, 7 y 8 se presentan las correlaciones entre las variables de producción, calidad de leche, estado corporal y peso para la totalidad de las vacas (Tabla 5) y para los distintos biotipos, a saber Holando, Jersey y cruza (Tablas 6, 7 y 8 ). Se consideran cuatro niveles de correlación de acuerdo con el valor absoluto del coeficiente de correlación de Pearson: no significativa $(0 \leq|\mathrm{r}|<0.1)$, baja $(0.1$ $\leq|\mathrm{r}|<0.3)$, media $(0.3 \leq|\mathrm{r}|<0.5)$ y alta $(0.5 \leq$ $|\mathrm{r}| \leq 1)$.

La correlación entre el estado al parto y la variación de estado con la producción de leche, aparece con mayor expresión en el biotipo Holando (Tabla 6) que en las vacas Jersey y cruza (Tablas 7 y 8); lo cual parece querer decir que hay más dependencia para producir leche de las reservas corporales en la raza Holando que en las otras razas. Esto parece evidenciarse también en las medias de las variables de los biotipos según nivel de Estado Corporal donde las Holando, que producen más leche, paren con estado al parto 3.38 y pierden más estado que los otros biotipos ( 0.74 unidades).

Las correlaciones de estado al parto y variación de estado con porcentaje de grasa son bajas con Estado Corporal y muy bajas y negativas con variación de estado para todas las vacas. Por tanto, surge la duda de para donde van las 
reservas de grasa del cuerpo que se gastan en dar leche, pero que no inciden en el porcentaje de grasa, como es citado por varios estudios (Broster, 1971).

En cambio la correlación de variación de estado con producción de leche es media (casi 0.5) en Holando y 0.46 en cruza. Debemos suponer, entonces, que la pérdida de reservas va para producción de leche. Si la mayor variación de estado con lleva mayor producción de leche, puede haber dilución de sólidos (por mayor producción de leche) y así, en parte explicarse que la mayor movilización de reservas no implique mayor porcentaje grasa. Tal vez asociado a esto, se puede observar que son negativas y/o bajas las correlaciones entre variación de estado y porcentaje de proteína y grasa (Tablas 5, 6, 7 y 8). También las correlaciones entre producción de leche $\mathrm{y}$ porcentaje de grasa y proteína son bajas y/o negativas; lo que significa que por el efecto dilución, a mayor producción de litros de leche, menor es el porcentaje de grasa y proteína.
La producción de grasa diaria presenta alta correlación con la producción de leche (0.789) y media con porcentaje de grasa (0.312) en todas las vacas (Tabla 5); en Holando es más alta la correlación con producción de leche $(0.856)$ y negativa y baja con porcentaje de Grasa (-0.212); en Jersey en cambio la correlación con producción de leche $(0.676)$ es menor que para todas las vacas y menor que para el Holando. También para el Jersey es mayor la correlación de kilos de grasa diaria con el porcentaje de grasa (0.461). Se evidencia entonces un comportamiento diferente entre ambas razas: para producir grasa láctea, el Holando dependería casi exclusivamente de producción de leche en cambio la Jersey apela a ambas variables (producción de leche y porcentaje grasa). También las reservas corporales al parto, afectan la variable producción de grasa (Broster, 1971).

Tabla 5. Tabla de correlaciones entre variables productivas, calidad de leche, estado corporal y peso para la totalidad de las vacas

\begin{tabular}{|c|c|c|c|c|c|c|c|c|c|c|c|c|}
\hline $\mathbf{R}$ & ECP & EC1 & EC2 & VAREC & PESO & PL & Gr & Pr & KgGrD & KgPrD & GrGrPV & GrPrPV \\
\hline ECP & 1.000 & & & & & & & & & & & \\
\hline EC1 & 0.320 & 1.000 & & & & & & & & & & \\
\hline EC2 & 0.309 & 0.324 & 1.000 & & & & & & & & & \\
\hline VAREC & 0.547 & -0.298 & -0.390 & 1.000 & & & & & & & & \\
\hline PESO & 0.074 & 0.044 & 0.033 & 0.016 & 1.000 & & & & & & & \\
\hline PL & 0.207 & -0.198 & -0.124 & 0.369 & 0.468 & 1.000 & & & & & & \\
\hline Gr & 0.211 & 0.131 & 0.146 & 0.009 & -0.400 & -0.254 & 1.000 & & & & & \\
\hline Pr & -0.033 & 0.164 & 0.212 & -0.185 & -0.276 & -0.400 & 0.484 & 1.000 & & & & \\
\hline KgGrD & 0.344 & -0.092 & -0.021 & 0.338 & 0.258 & 0.789 & 0.312 & -0.122 & 1.000 & & & \\
\hline KgPrD & 0.223 & -0.157 & -0.066 & 0.338 & 0.415 & 0.939 & -0.137 & -0.145 & 0.857 & 1.000 & & \\
\hline GrGrPV & 0.328 & -0.106 & -0.133 & 0.408 & -0.446 & 0.396 & 0.520 & 0.076 & 0.714 & 0.474 & 1.000 & \\
\hline GrPrPV & 0.189 & -0.197 & -0.224 & 0.419 & -0.390 & 0.545 & 0.138 & 0.112 & 0.632 & 0.644 & 0.874 & 1.000 \\
\hline
\end{tabular}

Referencias: PL - producción media de leche, Gr - porcentaje de grasa, Pr - proteína, KgGrD - producción diaria de grasa, KgPrD producción diaria de proteína, GrGrPV y GrPrPV - gramos de grasa y proteína por kg de peso, ECP- estado corporal al parto y en lactancia temprana y tardía (EC1, EC2), VAREC - diferencia entre ECP y EC1 o variación de estado 
Tabla 6. Tabla de correlaciones entre variables productivas, calidad de leche, estado corporal y peso para las

\begin{tabular}{|c|c|c|c|c|c|c|c|c|c|c|c|c|c|}
\hline $\mathbf{R}$ & ECP & EC1 & EC2 & VAREC & PESO & PL & CS & $\mathbf{G r}$ & Pr & KgGrD & KgPrD & GrGrPV & GrPrPV \\
\hline ECP & 1.000 & & & & & & & & & & & & \\
\hline EC1 & 0.366 & 1.000 & & & & & & & & & & & \\
\hline EC2 & 0.266 & 0.372 & 1.000 & & & & & & & & & & \\
\hline VAREC & 0.626 & -0.112 & -0.254 & 1.000 & & & & & & & & & \\
\hline PESO & 0.320 & -0.032 & 0.084 & 0.160 & 1.000 & & & & & & & & \\
\hline PL & 0.397 & -0.285 & -0.083 & 0.495 & 0.450 & 1.000 & & & & & & & \\
\hline CS & -0.182 & -0.002 & 0.096 & -0.249 & 0.120 & -0.130 & 1.000 & & & & & & \\
\hline Gr & 0.141 & 0.343 & 0.216 & -0.137 & -0.349 & -0.280 & -0.021 & 1.000 & & & & & \\
\hline $\operatorname{Pr}$ & -0.090 & 0.480 & 0.310 & -0.330 & -0.192 & -0.373 & 0.103 & 0.554 & 1.000 & & & & \\
\hline KgGrD & 0.479 & -0.103 & -0.075 & 0.440 & 0.353 & 0.856 & -0.212 & 0.212 & -0.170 & 1.000 & & & \\
\hline KgPrD & 0.399 & -0.160 & -0.125 & 0.450 & 0.464 & 0.966 & -0.165 & -0.152 & -0.185 & 0.887 & 1.000 & & \\
\hline GrGrPV & 0.329 & -0.205 & -0.146 & 0.519 & -0.090 & 0.697 & -0.325 & 0.254 & -0.179 & 0.891 & 0.712 & 1.000 & \\
\hline GrPrPV & 0.218 & -0.221 & -0.207 & 0.470 & 0.042 & 0.852 & -0.279 & -0.139 & -0.113 & 0.833 & 0.895 & 0.865 & 1.000 \\
\hline
\end{tabular}

Referencias: ídem Tabla 5.

Tabla 7. Tabla de correlaciones entre variables productivas, calidad de leche, estado corporal y peso para las vacas Jersey

\begin{tabular}{|c|c|c|c|c|c|c|c|c|c|c|c|c|c|}
\hline $\mathbf{R}$ & ECP & EC1 & EC2 & VAREC & PESO & PL & CS & Gr & Pr & KgGrD & KgPrD & GrGrPV & GrPrPV \\
\hline ECP & 1.000 & & & & & & & & & & & & \\
\hline EC1 & 0.370 & 1.000 & & & & & & & & & & & \\
\hline EC2 & 0.197 & 0.308 & 1.000 & & & & & & & & & & \\
\hline VAREC & 0.554 & -0.579 & -0.102 & 1.000 & & & & & & & & & \\
\hline PESO & -0.176 & -0.101 & 0.209 & -0.104 & 1.000 & & & & & & & & \\
\hline PL & 0.157 & 0.006 & -0.128 & 0.136 & -0.122 & 1.000 & & & & & & & \\
\hline CS & 0.103 & -0.107 & 0.058 & 0.115 & 0.218 & 0.097 & 1.000 & & & & & & \\
\hline Gr & 0.174 & 0.043 & 0.018 & 0.186 & -0.154 & -0.209 & 0.191 & 1.000 & & & & & \\
\hline Pr & -0.080 & -0.112 & 0.207 & -0.057 & 0.205 & -0.350 & 0.142 & 0.322 & 1.000 & & & & \\
\hline KgGrD & 0.258 & 0.009 & -0.067 & 0.241 & -0.261 & 0.676 & 0.180 & 0.464 & -0.031 & 1.000 & & & \\
\hline KgPrD & 0.128 & -0.067 & 0.009 & 0.125 & -0.118 & 0.875 & 0.116 & -0.103 & 0.022 & 0.802 & 1.000 & & \\
\hline GrGrPV & 0.380 & 0.116 & -0.262 & 0.219 & -0.573 & 0.611 & 0.027 & 0.451 & -0.122 & 0.919 & 0.629 & 1.000 & \\
\hline GrPrPV & 0.238 & 0.006 & -0.229 & 0.237 & -0.554 & 0.807 & 0.138 & -0.043 & -0.045 & 0.753 & 0.866 & 0.832 & 1.000 \\
\hline
\end{tabular}

Referencias: ídem Tabla 5. 
Tabla 8. Tabla de correlaciones entre variables productivas, calidad de leche, estado corporal y peso para las

\begin{tabular}{|c|c|c|c|c|c|c|c|c|c|c|c|c|c|}
\hline $\mathbf{R}$ & ECP & EC1 & EC2 & VAREC & PESO & PL & CS & $\mathbf{G r}$ & Pr & KgGrD & KgPrD & GrGrPV & GrPrPV \\
\hline ECP & 1.000 & & & & & & & & & & & & \\
\hline EC1 & 0.260 & 1.000 & & & & & & & & & & & \\
\hline EC2 & 0.355 & 0.325 & 1.000 & & & & & & & & & & \\
\hline VAREC & 0.480 & -0.354 & -0.649 & 1.000 & & & & & & & & & \\
\hline PESO & 0.121 & 0.137 & 0.064 & 0.019 & 1.000 & & & & & & & & \\
\hline PL & 0.161 & -0.349 & -0.364 & 0.463 & 0.481 & 1.000 & & & & & & & \\
\hline CS & 0.033 & 0.006 & 0.051 & 0.004 & 0.101 & -0.245 & 1.000 & & & & & & \\
\hline Gr & 0.189 & 0.212 & 0.358 & -0.106 & -0.097 & 0.020 & -0.120 & 1.000 & & & & & \\
\hline $\operatorname{Pr}$ & -0.190 & 0.245 & 0.276 & -0.325 & 0.071 & -0.155 & -0.004 & 0.415 & 1.000 & & & & \\
\hline KgGrD & 0.270 & -0.203 & -0.050 & 0.343 & 0.387 & 0.871 & -0.257 & 0.470 & -0.004 & 1.000 & & & \\
\hline KgPrD & 0.138 & -0.299 & -0.248 & 0.373 & 0.483 & 0.961 & -0.237 & 0.114 & 0.062 & 0.889 & 1.000 & & \\
\hline GrGrPV & 0.244 & -0.209 & -0.127 & 0.388 & 0.058 & 0.770 & -0.292 & 0.476 & -0.053 & 0.938 & 0.786 & 1.000 & \\
\hline GrPrPV & 0.084 & -0.375 & -0.418 & 0.426 & 0.109 & 0.885 & -0.305 & 0.063 & -0.008 & 0.816 & 0.917 & 0.862 & 1.000 \\
\hline
\end{tabular}

Referencias: ídem Tabla 5.

Analizando la relación del estado corporal al parto y la movilización de reservas pos parto (VAREC) con la producción de grasa diaria, vemos que presenta correlación media con estado al parto de 0.344 y con la variación de estado de 0.338. Los valores son mayores en Holando (0.479 y 0.440 para estado al parto y variación de estado, respectivamente) y estas correlaciones son bajas para Jersey y evidencian un comportamiento diferente de ambas razas en relación a esta variable de kilos de grasa diaria. La otra variable que afecta el porcentaje y producción de grasa es la alimentación.

En relación a la producción de proteína diaria, es necesario mencionar que esta variable debería depender, en gran medida, de la producción de leche $\mathrm{y}$ del porcentaje de proteína. Por eso, interesa en este estudio la correlación muy alta de la producción diaria de proteína con producción de leche. Estas correlaciones son casi iguales o superiores a 0.9. En cambio, las correlaciones entre kilos de proteína diaria y porcentaje de proteína son muy bajas e incluso negativas en el rodeo general, tanto como en el Holando y son muy bajas en Jersey y cruza. Parece claro que es necesario, para producir mucha proteína láctea (variable principal del valor de la leche en Uruguay), lograr mucha producción de leche. Las otras variables que afectan el porcentaje de proteína son la genética y la alimentación.

Las variables de producción de grasa y proteína en relación al peso vivo (GrGrPV y GrPrPV) presentan correlaciones medias y negativas con peso vivo (-0.446 y -0.390). Las correlaciones con peso vivo son diferentes según los biotipos: en Jersey son negativas y altas lo que sugiere que a menor peso hay más gramos de proteína $\mathrm{y}$ grasa por kg de peso; en cruzas son medias y positivas y en Holando son muy bajas. Las correlaciones de gramos de grasa y proteína por kilo de peso vivo con variación de estado (VAREC) son para Holando medias a altas, para Jersey bajas y para cruza medias. Esto parece evidenciar que el Holando depende más de tener que mover reservas para producir leche y 
sólidos. La correlación de proteína y grasa en relación al peso vivo con producción de leche y las respectivas producciones diarias de ambos sólidos (producción diaria de proteína y grasa, Tablas 6, 7 y 8) es alta para todos los biotipos, evidenciando la asociación entre estas variables.

\subsection{Análisis de comparación de medias entre variables productivas agrupadas por nivel de estado al parto y por biotipo.}

En las Tablas 9 y 10, se presentan la comparación de medias de las variables productivas que agrupan las vacas por niveles de estado corporal al parto: bajo ( 2 y 2.5), medio (2.75 a 3) y alto (más de 3) y por biotipo según sean Holando, Jersey y cruzas.

El peso es tomado, en el presente estudio, en lactancia temprana-media, que es donde las vacas, en general, tienen menor estado; y por tanto no es por más estado al parto que hay más peso (recordemos que una unidad de estado corresponde en Holando a $50 \mathrm{~kg}$ de peso, Edmonsond et al, 1989). Debe haber más producción de leche por más peso, dado que el aumento de producción de origen genético, ha llevado al aumento de tamaño en las vacas (Buckley et al, 2000). La correlación en Holando y cruza es media a alta entre producción Lechera y peso, pero en Jersey es baja y negativa (Tablas 6, 7 y 8). Una posible explicación es que el Holando es más grande porque viene de selección genética por litros y esto implica mayor tamaño; esta asociación entre mayor peso y producción de leche es evidente en este estudio en la Tabla 9, que observan niveles medio y alto de estado al parto. El Jersey es capaz de producir bastantes litros con menor tamaño o peso, especialmente si hay origen genético neozelandés que es de menor porte.

En la Tabla 9, se ven las diferencias entre los grupos de estado al parto y los biotipos: la producción diaria de proteína es estadísticamente menor en Jersey en los grupos de medio y alto nivel de estado al parto, en relación al Holando. También es menor en Jersey frente a la cruza en el nivel alto de estado corporal al parto. La producción diaria de grasa es estadísticamente menor en Jersey, en el grupo de alto nivel de estado al parto, en relación al Holando y las cruzas.

Tabla 9. Comparación de medias de variables productivas y composición de leche, agrupadas por nivel de estado al parto y por biotipo

\begin{tabular}{cccccccccc}
\hline Nivel ECP & Biotipo & Peso & PL & Gr & Pr & KgGrD & KgPrD & GrGrPV & GrGrPV \\
\hline \multirow{2}{*}{ BAJO: 2 Y 2.25 } & HOLAN & $545 \mathrm{a}$ & $17.1 \mathrm{a}$ & $3.9 \mathrm{~b}$ & $3.5 \mathrm{~b}$ & $0.66 \mathrm{a}$ & $0.60 \mathrm{a}$ & $1.29 \mathrm{a}$ & $1.17 \mathrm{a}$ \\
& JER+CRU & $485 \mathrm{a}$ & $15.7 \mathrm{a}$ & $4.8 \mathrm{a}$ & $4.1 \mathrm{a}$ & $0.72 \mathrm{a}$ & $0.62 \mathrm{a}$ & $1.43 \mathrm{a}$ & $1.33 \mathrm{a}$ \\
\hline \multirow{3}{*}{ MEDIO: $2.5 \mathrm{~A} \mathrm{3}$} & HOLAN & $520 \mathrm{a}$ & $20.1 \mathrm{a}$ & $3.89 \mathrm{~b}$ & $3.57 \mathrm{~b}$ & $0.76 \mathrm{a}$ & $0.70 \mathrm{a}$ & $1.43 \mathrm{a}$ & $1.36 \mathrm{a}$ \\
& JERSEY & $364 \mathrm{~b}$ & $13.8 \mathrm{~b}$ & $4.72 \mathrm{a}$ & $3.99 \mathrm{a}$ & $0.65 \mathrm{a}$ & $0.55 \mathrm{~b}$ & $1.83 \mathrm{a}$ & $1.51 \mathrm{a}$ \\
& CRUZA & $480 \mathrm{a}$ & $16.8 \mathrm{ab}$ & $4.63 \mathrm{a}$ & $3.82 \mathrm{a}$ & $0.75 \mathrm{a}$ & $0.62 \mathrm{ab}$ & $1.59 \mathrm{a}$ & $1.33 \mathrm{a}$ \\
\hline \multirow{3}{*}{ ALTO: 3.25 Y MAS } & HOLAN & $585 \mathrm{a}$ & $25.5 \mathrm{a}$ & $4.3 \mathrm{~b}$ & $3.5 \mathrm{~b}$ & $1.07 \mathrm{a}$ & $0.88 \mathrm{a}$ & $1.83 \mathrm{a}$ & $1.49 \mathrm{a}$ \\
& JERSEY & $357 \mathrm{c}$ & $15.5 \mathrm{c}$ & $5.1 \mathrm{a}$ & $3.99 \mathrm{a}$ & $0.78 \mathrm{~b}$ & $0.61 \mathrm{~b}$ & $2.25 \mathrm{a}$ & $1.73 \mathrm{a}$ \\
& CRUZA & $519 \mathrm{~b}$ & $21.7 \mathrm{~b}$ & $4.5 \mathrm{~b}$ & $3.7 \mathrm{a}$ & $0.99 \mathrm{a}$ & $0.80 \mathrm{a}$ & $1.96 \mathrm{a}$ & $1.57 \mathrm{a}$ \\
\hline
\end{tabular}

Letras diferentes significan diferencias estadísticas $\mathrm{P}$ menor 0.05

Referencias: PESO - peso vivo, PL - producción media de leche, Gr - porcentaje de grasa, Pr - porcentaje de proteína, KgGrD producción diaria de grasa, KgPrD producción diaria de proteína, GrGrPV y GrPrPV - gramos de grasa y proteína por kg de peso. Nota: en el nivel bajo de ECP al haber pocas vacas se agruparon las holando por un lado y las jersey y cruzas juntas en otro grupo. 
Tabla 10. Comparación de medias de variables productivas y composición de leche, agrupadas por biotipo

\begin{tabular}{ccccccccc} 
Biotipo & Peso & PL & Gr & Pr & KgGrD & KgPrD & GrGrPV & GrPrPV \\
\hline HOLAN & $534 \mathrm{a}$ & $18.6 \mathrm{a}$ & $4.04 \mathrm{c}$ & $3.59 \mathrm{~b}$ & $0.75 \mathrm{~b}$ & $0.67 \mathrm{a}$ & $1.48 \mathrm{c}$ & $1.32 \mathrm{~b}$ \\
JERSEY & $340 \mathrm{c}$ & $14.9 \mathrm{~b}$ & $4.91 \mathrm{a}$ & $3.96 \mathrm{a}$ & $0.699 \mathrm{~b}$ & $0.57 \mathrm{~b}$ & $2.25 \mathrm{a}$ & $1.799 \mathrm{a}$ \\
CRUZA & $505 \mathrm{~b}$ & $19.2 \mathrm{a}$ & $4.43 \mathrm{~b}$ & $3.68 \mathrm{~b}$ & $0.86 \mathrm{a}$ & $0.71 \mathrm{a}$ & $1.79 \mathrm{~b}$ & $1.46 \mathrm{~b}$ \\
\hline
\end{tabular}

Letras diferentes significan diferencias estadísticas P menor 0.05 Referencias: ídem Tabla 9.

Las variables de producción de grasa y proteína en relación al peso vivo presentan correlaciones medias y negativas con peso vivo (tablas 6 a 8); lo que adelanta el hecho de que el ganado Jersey, de menor tamaño, produce más sólidos en relación al $\mathrm{kg}$ de peso vivo como se observa en la Tabla 10. Las correlaciones con peso vivo son diferentes según los biotipos: en Jersey son negativas y altas lo que sugiere que a menor peso hay más gramos de proteína y grasa por $\mathrm{kg}$ de peso; en cruzas son medias y positivas y en Holando muy bajas. Las correlaciones de GrGrPV y GrPrPV con variación de estado (VAREC) son para Holando medias a altas, para Jersey bajas y para las cruza medias; esto sugiere que el Holando depende más de mover reservas para producir leche y sólidos.

Hay bastante más producción de leche en el grupo de alto estado al parto comparado con el nivel medio de ECP en todos los biotipos (Tabla 9), lo que puede hacer referencia al efecto de las reservas corporales en la producción de leche (Krall y Chilibroste, 2003). En el Holando, es media la correlación entre estado al parto y producción lechera, sin embargo, en las cruza y Jersey es baja esa correlación (Tablas 6, 7 y 8).

Cuando analizamos la Tabla 10, se comparan las variables en relación al biotipo parecen evidenciarse las diferencias entre biotipos: el mayor peso en el Holando es esperable, sin embargo, llama la atención la mayor producción de leche, grasa y proteína diaria en la cruza respecto al Holando (Comerón, 2003; Marini y Krall, 2006). Las razones de estos resultados pueden ser la muy buena performance productiva, número de animales y buena alimentación de la triple cruza del predio E, además del vigor híbrido de este ganado.

A su vez el origen genético dentro del Holando de estos tambos es mayoritariamente Neozelandés y Nacional, siendo solamente un $25 \%$ del total de dicha raza el de origen Norteamericano que es más productora de leche. Es esperable (González y Magofke, 2003; Krall et al, 2018) el mayor porcentaje de grasa y proteína del Jersey, así como, la mayor (estadísticamente) producción de sólidos en relación al peso vivo de esta raza en relación a los otros dos biotipos. Dado la menor producción de leche del Jersey, es esperable una menor producción diaria de grasa y proteína de este biotipo respecto al Holando y las cruzas como se observa en la Tabla 10. El mayor porcentaje de los sólidos en Jersey no permite en este caso equilibrar la producción diaria de sólidos de los otros dos biotipos que producen más leche.

Si se compara la cruza con el Holando es interesante visualizar que en los estados al parto medios y altos, (Tabla 9) los litros de leche son mayores en el segundo caso, pero no hay diferencias con la cruza en la producción diaria de los sólidos. Esto es diferente cuando comparamos la cruza con el Holando, sin 
dividir los grupos por estado al parto (Tabla 10), aquí la cruza produce algo más de litros de leche y más sólidos diarios (grasa diaria estadísticamente mayor y proteína diaria absolutamente mayor).

En el presente estudio, se observa en el Holando una caída de reservas corporales desde el estado al parto hasta las dos mediciones de estado corporal en lactancia (EC1, EC2) para los tres niveles de estado al parto que recuerda lo mencionado antes sobre la dependencia del Holando para producir mayor producción de leche (Blake y Custodio, 1984). El ajuste del alimento a los requerimientos es, en estos sistemas de producción, más difícil de realizar en la raza Holando que en la cruza o el Jersey, que son biotipos más rústicos y en los cuales se da una recuperación de reservas desde lactancia temprana hacia la media.

\section{CONCLUSIONES}

Las obvias diferencias entre sistemas y biotipos de producción existentes evidencian la necesidad de información apropiada para comprenderlos. Además, se debe prestar atención a lo económico y productivo a la hora de valorar estas opciones de sistema, por ejemplo, reducir o incrementar gastos tiene consecuencias productivas que deben ser medidas técnicamente con precisión y ser miradas en conjunto con las variables físicas (producción, reproducción, salud, longevidad).

Variables como reproducción, producción de leche y sólidos, alimentación y estado corporal determinadas con certeza, favorecen mejores evaluaciones del sistema y ganado utilizado.

El ajuste del alimento a los requerimientos es, en estos sistemas de producción, más difícil de realizar en la raza Holando que en la cruza o la Jersey, que son biotipos más rústicos y en los cuales se da una recuperación de reservas corporales, desde lactancia temprana hacia la media. El cruzamiento es una opción que implica aprovechar el vigor híbrido (puede ser beneficioso tanto para el producto leche como carne) y hay opciones diferentes en el sentido de la genética elegida. Un desafío importante es poder valorar adecuadamente la producción de sólidos en relación al peso vivo; esta variable pretende integrar los requerimientos de mantenimiento que en mucho dependen del peso vivo, así como la producción.

Hay diferentes estrategias de sistema como mayor gasto y producción (intensificación) o lo inverso. Pero esto debe tener un correlato con el biotipo elegido, la restricción del alimento utilizado debería acompañarse de la opción por biotipos más rústicos. En estos predios estudiados se dan ambas opciones (mayor y menor intensificación) y tal vez falta información para comprender totalmente situaciones como, por ejemplo, que pudiera ser que los predios de mayor intensificación se acompañaran de un mayor endeudamiento o que los de menor gasto apostaran a no generar deudas. Además, se hacen necesarios más trabajos que integren resultado económico, producción, reproducción, salud, sustentabilidad de los recursos humanos y naturales, permitirán mejores posibilidades de orientación hacia la sobrevivencia de los tambos en esta difícil coyuntura de la lechería exportadora uruguaya.

\section{Agradecimientos:}

Los autores agradecen a las familias productoras y sus trabajadores que gentilmente colaboraron en este trabajo. 


\section{Referencias}

ANDE (2020). https://www.ande.org.uy/beneficiarios/ item/desarrollo-de-un-sistema-de-informacion-parala-transferencia-tecnologica-y-extension-en-lacadena-lactea-de-la-region-litoral-bajo-un-nuevomodelo-de-articulacion-interinstitucional.html, Recuperado, diciembre 2020.

Bartaburu, D., Cesar R. (2016) Descenso de precios en la lechería uruguaya: una mirada participativa a la gestión predial. Revista Plan Agropecuario, 157. https://www.planagropecuario.org.uy/publicacione s/revista/R157/R_157_32.pdf.

Blake, R.W.; Custodio, A.A. (1984) Feed efficiency: a composite trait of dairy cattle. Journal of Dairy Science. 62:2075-2083.

Broster, W. (1971) The effect on milk yield of the cow of the level of feeding before calving. Journal of Dairy Science. V.33. (4), 253-270.

Buckley, F.; Dillon, P.; Rath, M.; Veerkamp, R.F. (2000) The relationship between genetic merit for yield and live weight, condition score, and energy balance of spring calving Holando-Friesian dairy cows on grass based systems of milk production. Journal of Dairy Science. 83:1878-1886.

Comeron, E. (2003) Efecto racial sobre la composición de la leche. INTA Rafaela. Merco láctea 2003.

Diario La Mañana (2020). https://www.xn--lamaana7za.uy/agro/para-los-tambos-la-unica-solucion-esbajar-los-costos-del-pais/, recuperado mayo, 2020.

Edmonson, A.J.; Lean, I.J.; Weaver, L.D.; Farver, T.; Webster, G. (1989) A body condition scoring chart for Holstein dairy cows. Journal of Dairy Science 72: 6878. doi: 10.3168/ jds.S0022-0302(89)79081-0.

DIEA (2020). Anuario Estadístico, Montevideo, Uruguay.
González, H.V.; Magofke, J.C. (2003) Cruzamientos y producción de leche. Instituto de Investigaciones Agropecuarias - Centro Regional de Investigación Remehue. Seria Actas $N^{\circ} 24$.

INALE (2020): https://www.inale.org/remision-enero2021-se-ubica-6-por-encima-de-la-del-mismo-mesdel-ano-2020/, recuperado, diciembre 2020.

INALE (2014) Presentación resultados preliminares Encuesta INALE. https:/www.inale.org/historico/ wp-content/uploads/.../Encuesta-lechera-2014.pdf, recuperado, enero 2021.

Krall, E.; Chilibroste P. (2003) Efecto de dos niveles de oferta de concentrado y el estado corporal al parto sobre la producción y la reproducción de ganado lechero. Revista de la SMVU.

Krall, E.; Saldanha, S.; Marquez, L. (2018) Comportamiento de vacas Holando, Jersey y sus cruzas con bajo nivel de suplementación y uso de campo natural y pasturas mejoradas. Congreso FEPALE Buenos Aires, Argentina.

Marini, P.; Krall, E. (2006). Evaluación de biotipos lecheros en sistemas de producción del litoral norte argentinouruguayo. XXXIV Jornadas Uruguayas de Buiatría. Paysandú, Uruguay.

Montosi, F.; Pravia, M.I.; Dighiero, A.; Porcile, V.; Gutierrez, D.; De Barbieri, I. (2013) Estimaciones indirectas de la cantidad de forraje para una mejor gestión de nuestras pasturas. Revista INIA, 34, 25-29

Rosa, A.L; Arbeletche, P. (2016) Caracterización de sistemas productivos de la cuenca lechera del Litoral Norte del Uruguay. Pre Congreso ALASRU (Asociación Latinoamericana de Sociología Rural). La Sociología rural en la encrucijada: vigencia de la cuestión agraria, actores sociales y modelos de desarrollo en la región. Santiago del Estero, Argentina. 